\title{
Tumor sólido pseudopapilar de páncreas, reporte de un caso
}

\author{
RENATO ACUÑA L. ${ }^{1}$, PATRICIO SOTO L. ${ }^{2}$, VANESSA MUÑOZ R. ${ }^{3}$, JAVIERA ACUÑA R. ${ }^{4}$ \\ 1. Cirujano Infantil, Jefe de Servicio de Cirugía Infantil, Hospital San Juan de Dios. \\ 2. Cirujano Infantil, Servicio de Cirugía Infantil, Hospital San Juan de Dios. \\ 3. Becada de Cirugía Pediátrica, Universidad de Chile. \\ 4. Alumna de Medicina, Servicio de Cirugía Infantil, Hospital San Juan de Dios.
}

\begin{abstract}
Pancreas pseudo-papillary tumor, a case-report

Pancreatic tumors are extremely rare, specially in children, where the pancreas pseudo-papillary tumor corresponds to $1 \%$ of primary pancreatic tumors, characterized by its low malignant potential. We report the case of a solid pancreas pseudo-papillary tumor in a 5 year-old female patient, presenting with prolonged abdominal pain. An abdominal ultrasonography and computed tomography were performed, showing 2 tumors at the pancreas head and tail, with normal tumor markers (alpha-fetoprotein, carcinoembryonic antigen, $\beta$-HCG). The surgical treatment consisted on distal pancreatectomy with tumor enucleation at the pancreas head and tail, afterwards presenting acute pancreatitis that was medically treated. The biopsy confirmed pancreas solid pseudo-papillary tumor. In the 22 months follow-up period, the patient has remained disease-free.

(Key words: pediatric pancreas tumor, pancreas solid pseudo-papillary tumor, distal pancreatectomy, conservative surgery)

Rev Chil Pediatr 2008; 79 (4): 404-408
\end{abstract}

\section{RESUMEN}

Objetivo: Las neoplasias de páncreas son muy infrecuentes, más aún en la edad pediátrica, y el tumor sólido pseudopapilar de páncreas corresponde alrededor del 1\% de los tumores primarios de páncreas, caracterizándose por su bajo potencial maligno. Reportamos el caso clínico de un tumor sólido pseudopapilar de páncreas en paciente pediátrico. La paciente es una niña de cinco años, la cual consultó por dolor abdominal prolongado, dentro de su estudio se realizó ecografía y escáner abdominal demostrando dos masas en cabeza y cola de páncreas, con marcadores tumorales (alfafetoproteína, antígeno carcinoembrionario, $\beta$-HCG) normales. Se realizó pancreatectomía distal y enucleación del tumor de cabeza de

Trabajo recibido el 04 de diciembre de 2007, devuelto para corregir el 11 de marzo de 2008, segunda versión el 04 de junio de 2008, aceptado para publicación el 10 de junio de 2008.

Correspondencia a:

Vanessa Muñoz Ramos

E-mail:Vanem75@yahoo.com 
páncreas; en postoperatorio evolucionó con pancreatitis de manejo médico. Biopsia confirmó tumor sólido pseudopapilar de páncreas. En seguimiento de 22 meses la paciente se ha mantenido libre de enfermedad.

Conclusión: Ante este raro tumor de páncreas se debe realizar un completo estudio preoperatorio y es recomendable la cirugía conservadora

(Palabras clave: tumor páncreas pediátrico, tumor sólido pseudopapilar, pancreatectomía distal, cirugía conservadora).

Rev Chil Pediatr 2008; 79 (4): 404-408

\section{Introducción}

El páncreas es un sitio extremadamente infrecuente de neoplasia en niños y adolescentes $^{1}$. De todos los tumores primarios de páncreas, el tumor sólido pseudopapilar corresponde sólo al 0,13 a $2,7 \%$ de los $\operatorname{casos}^{1-4}$. Desde 1959 se han reportado aproximadamente 500 casos de tumores sólido pseudopapilar de páncreas, de los cuales sólo 35 corresponden a casos pediátricos ${ }^{5}$. La mayoría de los tumores pancreáticos son malignos y tiene un mal pronóstico; sin embargo, el tumor sólido pseudopapilar tiene un bajo potencial maligno, que rara vez da metástasis ${ }^{2,6}$, describiéndose tasa de sobrevida de $90-95 \%$ a los 5 años ${ }^{6-8}$.

La forma de presentación más frecuente es dolor abdominal y/o masa abdominal $1^{1-4,7,8,10-12}$, se han descrito otros síntomas y signos los que incluyen dispepsia ${ }^{6,7}$, náuseas, vómitos ${ }^{12}$, amilasemia elevada ${ }^{7}$, ictericia ${ }^{1,6,13}$, o también, puede ser un hallazgo incidental ${ }^{2,6}$; otra forma de presentación es como abdomen agudo por hemoperitoneo $^{2,4,10,13}$. Se presenta característicamente en mujeres jóvenes entre la segunda y tercera década de la vida, siendo la relación hombre: mujer de $1: 9^{1,2,4,6,10,13}$

Debido al potencial de malignidad de estas lesiones (aproximadamente 12-15\% de los casos), la resección quirúrgica ha sido el estándar en el manejo de los tumores sólido pseudopapilar del páncreas ${ }^{3,4,7,12}$, sin embargo, la extensión de la resección varia, principalmente según la edad del paciente y la localización de la masa.

Se presenta el caso de una menor de cinco años con un tumor sólido pseudopapilar de páncreas tratado en nuestro hospital con cirugía conservadora.

\section{Caso clínico}

Niña de 5 años 8 meses, sin antecedentes mórbidos de importancia, la cual presentó dolor epigástrico de 16 meses de evolución, por este motivo había consultado en múltiples ocasiones en Servicio de Urgencia, siendo finalmente derivada a policlínico de gastroenterología infantil. Al examen físico sólo destacaba leve dolor epigástrico a la palpación, sin masas, ni visceromegalia. Se realizó ecografía abdominal que mostró masa sólida irregular con discreto aumento de la circulación entre bazo y riñón de 9 $\mathrm{cm}$. Haciendo cuerpo con la cola del páncreas. Se tomó nueva ecografía a los dos días, la cual mostró una masa hipoecogénica mal definida de $7 \times 7 \mathrm{~cm}$ con áreas anecogénicas y probables calcificaciones, planteando un posible origen en la glándula suprarrenal. Se realizó TAC de abdomen, el cual mostró, en relación a la cola del páncreas, una masa sólida de $10 \times 8$ $\mathrm{cm}$, que se refuerza con el medio de contraste en forma tardía y heterogénea, además, presenta calcificaciones puntiformes. Se aprecia otro nódulo de iguales características, en relación al proceso uncinado del páncreas que mide $3 \mathrm{~cm}$ de diámetro. Los resultados de los marcadores tumorales fueron: alfafetoproteína: 1,0 $\mathrm{ng} / \mathrm{ml}$, antígeno carcinoembrionario: $1,09 \mathrm{ng} / \mathrm{ml}$, $\beta$-HCG: $<0,5 \mathrm{mUI} / \mathrm{ml}$, todos en rangos normales.

\section{Cirugía}

Al octavo día se realizó laparotomía media, encontrando un tumor de $10 \times 15 \mathrm{~cm}$. En cola de páncreas, el cual presentaba cápsula y esta bien delimitado (figura 1), se realizó pancreatectomía distal, extirpando completamente este 


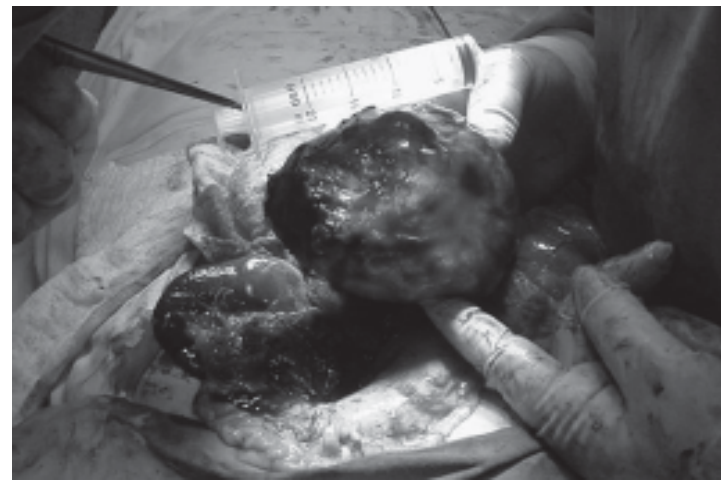

Figura 1.

tumor. El segundo tumor se localizó en cabeza de páncreas, midió $2 \times 2 \mathrm{~cm}$ y fue enucleado (figura 2), procedimiento en el cual no se comprometió la vía biliar. Se dejó un drenaje al lecho. La cirugía se realizó sin incidentes.

En el postoperatorio, evolucionó con pancreatitis, presentando al segundo día postoperatorio amilasemia sobre $1400 \mathrm{u} / 1$, se manejó médicamente, y la amilasemia disminuyó notoriamente a las 48 horas. La paciente se realimentó al $6^{\circ}$ día de la cirugía, y se fue de alta a los 13 días postoperatorios.

En la macroscopía de la biopsia se describe: masa de cola de páncreas de $10 \times 7,5 \times 6,5 \mathrm{~cm}$ y de la cabeza de páncreas de $3 \times 2,5 \times 2,3 \mathrm{~cm}$, ambas de superficie abollonada, color pardo amarillento y con pequeñas placas hemorrágicas. $\mathrm{Al}$ corte tienen aspecto heterogéneo conformado por lóbulos, zonas necrohemorrágicas, quistes de tamaño irregular y septos blanquecinos amarillentos irregulares. A la microscopía se observa, tumor sólido de células uniformes conformando estructuras pseudopapilares y focos de degeneración quística; las células con leve atipia nuclear de citoplasma eosinófilo y en sectores tienen citoplasma vacuolado, abundante áreas de necrosis y hemorragia, estroma fibrovascular hialino y mixoide, actividad mitótica prominente en la periferia de la cápsula fibrosa con microcalcificaciones y estructuras ductales. Se realizó, además, análisis inmunohistoquímico, cuyos resultados son positivos para $\alpha$ lantitripsina, $\alpha$ lantiquimiotripsina, vimentina y enolasa neuroespecífica (NSE). Se concluye, tumor sólido quístico del páncreas.

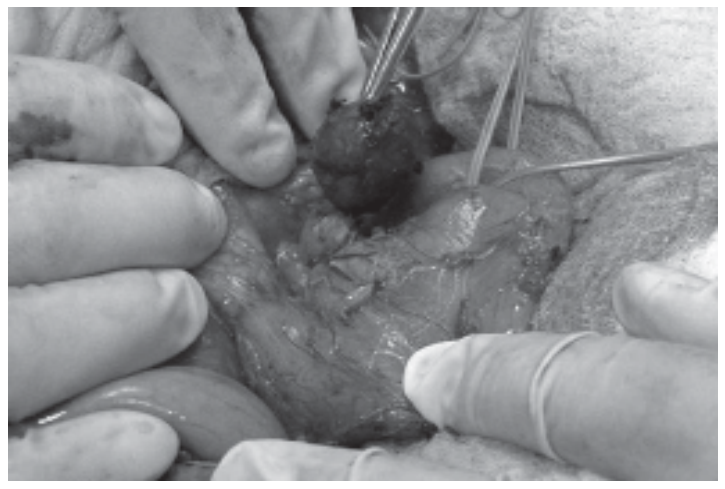

Figura 2.

La paciente tiene un seguimiento de 22 meses en los cuales se mantiene asintomática y con ecografías normales.

\section{Discusión}

El primer tumor sólido pseudopapilar (TSP) de páncreas fue descrito por Frantz en $1959^{14}$ y desde entonces se ha nominado con una variedad de términos, incluyendo tumor de Frantz, tumor sólido-quístico, tumor papilar quístico, neoplasia epitelial sólido y papilar, neoplasia papilar quística y neoplasia epitelial papilar quística ${ }^{1,6,13}$

El TSP afecta predominantemente a mujeres $(80-90 \%)$ y más del $50 \%$ ocurre en la segunda década de la vida, es inusual en la primera década ${ }^{1,3,4,6}$, por lo que llama la atención en el caso presentado que corresponde a una menor de 5 años de edad, siendo que el promedio de edad descrito oscila entre 25 y 35 años $1,2,4,6,10,13$. Choi presentó 23 casos de los cuales el menor tenía 10 años $^{7}$ y Shorter describe 17 casos de los cuales sólo cuatro tienen 5 años o menos ${ }^{1}$. La forma de presentación en el caso descrito, dolor abdominal epigástrico, concuerda con la clínica habitual de este tipo de tumores; sin embargo, hubo retraso en el diagnóstico, recién después de 16 meses de iniciado los síntomas, en parte por lo inusual de esta patología y la baja sospecha por parte de los médicos que la atendieron, teniendo en consideración que el síntoma dolor es muy inespecífico; y por otro lado, debido a que la paciente sólo consultaba en Servicio de Urgencia, lo cual 
probablemente determinó, la demora en la correcta derivación para su estudio.

El diagnóstico diferencial de TSP de páncreas incluye cualquier enfermedad pancreática sólida o quística, tales como: tumor quístico mucinoso, adenoma microquístico, tumor de células de los islotes, carcinoma de células acinares, cistoadenocarcinoma, adenocarcinoma ductal, pseudoquiste inflamatorio, tumor secretor de mucus, pancreatoblastoma, tumores vasculares tipo hemangioma y neoplasia pancreática endocrina (benigna y maligna) ${ }^{1,2}$, sin embargo, durante la niñez prácticamente se restringe al pancreatoblastoma ${ }^{4}$. Por esto, ante un tumor de páncreas con expansión local, con cápsula bien definida, sin signos de metástasis, en una mujer adolescente, se debe considerar altamente sospechoso de $\mathrm{TSP}^{4}$.

Las características radiológicas del TSP es muy importante para establecer el diagnóstico exacto antes de la cirugía. Las herramientas típicas usadas para diagnosticarlo son ecografía y TAC abdominal, en los cuales el TSP aparece como una masa sólida bien demarcada. En la ecografía usualmente presenta ecogenicidad heterogénea, algunas veces contiene áreas quísticas hipoecogénicas, y esta masa está en o adyacente al páncreas ${ }^{2,4,7,15}$. El escáner revela una masa retroperitoneal grande bien encapsulada y circunscrita, con centro heterogéneo, además de desplazamiento de las estructuras cercanas $^{2,4}$. La necrosis focal es común en este tumor y puede tener calcificaciones ${ }^{1}$. En nuestra paciente las ecografías describieron una masa irregular heterogénea con calcificaciones, pero no fue clara en definir su localización exacta, por otro lado, el escáner definió con bastante exactitud las características de las lesiones pancreáticas. Los exámenes de laboratorio suelen ser normales al igual que los marcadores tumorales (CA19,9, CA125, CEA, alfafetoproteína) $)^{1,6}$.

Macroscópicamente, el TSP es un gran tumor sólido, esférico bien demarcado, a menudo rodeado por una cápsula y solo rara vez invade órganos adyacentes; usualmente mide alrededor de 8 a $10 \mathrm{~cm}$. Al corte las superficies muestran grandes áreas de hemorragia alternando con degeneración pseudoquística ${ }^{2,4,6,7,12}$. La histología nos mostrara estructuras sólidas y pseudopapilares, intensa vacularización, abundante degeneración celular ${ }^{4}$, la apariencia histológica es muy distintiva y es considerada diagnóstica $^{2,4,7}$. Destaca en este caso la presencia de dos tumores en el páncreas, ya que usualmente son tumores solitarios localizados en páncreas $^{6}$, aunque se han descrito presentaciones multicéntricas o extrapancréaticas (en mesocolon, retroperitoneo, omento o hígado) ${ }^{6}$. El predominio de localización del TSP es en la cabeza de páncreas, seguido por la cola y menos frecuente en el cuerpo, Wang et al, reportaron que la distribución del tumor es similar en todos los grupos etarios ${ }^{4,9,12}$.

En cuanto a la inmunohistoquímica, es invaluable para realizar diagnóstico diferencial. Los TSP exhiben fuerte tinción para vimentina y para NSE, mientras que la inmunotinción para marcadores epiteliales suele estar ausente. La tinción para $\alpha$ lantitripsina y $\alpha$ lantiquimiotripsina muestran un patrón característico: células tumorales fuertemente positivas, diseminadas en pequeños grupos dentro de un fondo de células tumorales negativas ${ }^{3,6}$, en el caso presentado el tumor fue positivo para estos cuatro marcadores inmunohistoquímicos, lo cual confirma el diagnóstico de tumor sólido pseudopapilar.

En cuanto al tratamiento del TSP se ha descrito: pancreatectomía total, pancreatodoudenectomía, pancreatectomía distal, asociados o no, a esplenectomía o a disección sistemática de nódulos linfáticos ${ }^{4,7,8,12,13}$. La pancreaticoduodenectomía es comúnmente usada para resecar TSP localizado en la cabeza pancreática, mientras que la pancreatectomía distal es usada para los localizados en la cola. La esplenectomía es utilizada para tumor que afecta el hilio esplénico. Recientemente se ha utilizado la simple enucleación o la pancreatectomía distal laparoscópica ${ }^{7,13}$. La estrategia de tratamiento en pediatría del TSP es una completa resección con preservación del máximo de tejido pancreático posible. La resección local es por lo tanto la terapia de elección ${ }^{4}$, esto se basa, en que el 95\% de los pacientes con enfermedad restringida al páncreas son sanados por completo con la resección quirúrgica ${ }^{1}$. Siguiendo este criterio, en la paciente descrita se resecaron ambos tumores, conservando el máximo de tejido 
pancreático; con lo que ha presentado una buena evolución, actualmente sin recidiva. Destaca una revisión de la literatura, la cual encontró, que bajo los 20 años los pacientes tienen el doble de posibilidades de que se les realice una resección local ${ }^{3,9}$.

Nuestra paciente presentó una pancreatitis en el postoperatorio inmediato, la cual se manifestó por el alza en la amilasemia, la cual revirtió rápidamente, con medidas generales. Hay que destacar que diversas cirugías abdominales pueden acompañarse de una discreta hiperamilasemia sin otra evidencia de pancreatitis aguda. La pancreatitis aguda post quirúrgica ocurre por compromiso directo del páncreas o de su irrigación, en cirugías extrabiliares, pancreáticas y en trauma, y/o por compromiso obstructivo del sistema excretor pancreático, en cirugías biliares o post-colangiopancreatografía retrógrada endoscópica ${ }^{16,17}$.

Se ha reportado sobrevida del $90-95 \%$ a los 5 años. La incidencia de metástasis reportada es de 12 a $15 \%$ de los casos, estando la mayoría presentes al momento del diagnóstico ${ }^{1,6,7,9,12}$, las metástasis ocurren a hígado y peritoneo, rara vez a linfonodos ${ }^{6}$.

Como conclusión los tumores malignos de páncreas son muy infrecuentes en niños y adolescentes, por lo que se requiere un alto índice de sospecha, para poder diagnosticarlos en forma en precoz. Ante una masa pancreática, se debe estudiar con imágenes, las que incluye ecografía y scanner, de esta forma tendremos una buena aproximación diagnóstica preoperatoria. Si se trata de a un tumor de páncreas bien delimitado, sin metástasis, en un paciente pediátrico, lo más probable es que corresponda a un tumor sólido pseudopapilar; y una vez hecho este diagnóstico, se sugiere realizar una cirugía conservadora, preservando el máximo de tejido pancreático, la cual será curativa y permitirá una alta sobrevida a largo plazo libre de enfermedad.

\section{Referencias}

1.- Shorter N, Glick R, Klimstra D, Brennan M, Laquaglia K: Malignant pancreatic tumors in childhood and adolescence: the memorial sloan-kettering experience, 1967 to present. J Pediatr Surg 37: 887-92.
2.- Huang HL, Shih SC, Chang WS, Wang TE, Chen MJ, Chan YJ: Solid-pseudopapillary tumor of the pancreas: clinical experience and literature review. World J Gastroenterol 2005; 11 (9): 1403-9.

3.- Nadler EP, Novikov A, Landzberg B, et al: The use of endoscopic ultrasound in the diagnosis of solid pseudopapillary tumors of the pancreas in children. J Pediatr Surg 2002; 37 (9): 1370-3.

4.- Raffel A, Cupisti K, Krausch M, et al: Therapeutic strategy of papillary cystic and solid neoplasm (pcsn):a rare non-endocrine tumor of the pancreas in children. Surgical Oncology 2004; 13: 1-6

5.- Kempski HM, Austin N, Chatters SJ, et al: Previously unidentified complex cytogenetic changes found in a pediatric case of solid-pseudopapillary neoplasm of the pancreas. Cancer Genetics and Cytogenetics 2006; 164: 54-60.

6.- Adamthwaite J, Verbeke C, Stringer M, Guillou P, Menon $K$ : Solid pseudopapillary tumour of the pancreas: diverse presentation, outcome and histology. Jop. J Pancreas 2006; 7 (6): 635-42.

7.- Choi SH, Kim S, Oh JT, Park J, Seo J, Lee S: Solid pseudopapillary tumor of the pancreas: a multicenter study of 23 pediatric cases. J Pediatric Surg 2006; 41: 1992-5.

8.- Rebhandl W, Felberbauer FX, Puig S, et al: Solidpseudopapillary tumor of the pancreas (frantz tumor) in children: report of four cases and review of the literature. J Surg Oncol 2001; 76: 289-96.

9.- Wang KS, Albanese C, Dada F, et al: Papillary cystic neoplasm of the pancreas: a report of three pediatric cases and literature review. J Pediatr Surg 1998; 33: $842-5$

10.- Karatag O, Yenice G, Ozkurt H, Basak M, Basaran C, Yilmaz B: A case of solid pseudopapillary tumor of the pancreas. World J Gastroenterol 2006; 12 (38): 6239-43

11.- Asano T, Seya T, Tanaka N, Ooaki Y, Fujino O: A 13-year-old girl with a preoperatively diagnosed solid cystic tumor of the pancreas. J Nippon Med Sch 2006; 73: $231-4$

12.- Chen X, Zhou GW, Zhou HJ, Peng CH, Li HW: Diagnosis and treatment of solid-pseudopapillary tumors of the pancreas. Hepatobiliary Pancreat Dis Int 2005; 4 (3): 456-9.

13.- Zhang $H$, Liang $T$, Wang $W$, Shen $Y$, Ren $G$, Zheng $S$ : diagnosis and treatment of solid-pseudopapillary tumor of the pancreas. Hepatobiliary Pancreat Dis Int 2006; 5 (3): 454-8.

14.- Frantz VK: Tumors of the pancreas. In: atlas of tumor pathology, 1 st series. Washington, DC, USA: us armed forces institute of pathology 1959; 32-3.

15.- Wunsch LP, Flemming $P$, Werner $U$, et al: Diagnosis and treatment of papillary cystic tumor of the pancreas in children. Eur J Pediatr Surg 1997; 7: 45-7.

16.- Reber H: Páncreas. En: Schwartz. Principios de cirugía. Séptima edición. Editorial Mcgraw-Hill Interamericana $1998 ; 1563-70$

17.- Pancreatitis aguda. Manual de patología quirúrgica, Pontificia Universidad Católica de Chile, escuela de medicina. www.escuela.med.puc.cl/paginas/publicaciones/patolquir/patol quir_015.htlm. Http://escuela.med. puc.cl/publ/manualcirugia/indice.htm 